Artículo

\title{
Comportamiento agronómico y fisiológico de maíces nativos del sureste de México
}

\author{
Luis Filipe Conceição dos Santos ${ }^{1}$ \\ René Garruña ${ }^{1 \S}$ \\ Rubén H. Andueza-Noh ${ }^{1}$ \\ Luis Latournerie-Moreno ${ }^{1}$ \\ Javier O. Mijangos-Cortés ${ }^{2}$ \\ Agustín Pineda-Doporto ${ }^{3}$
}

${ }^{1}$ División de Estudios de Posgrado e Investigación-Instituto Tecnológico de Conkal. Avenida Tecnológico s/n, Conkal, Yucatán, México. CP. 97345. Tel. 999 9124135. (luisdaconceicao@itconkal.edu.mx; r_ andueza81@hotmail.com; luis.latournerie@itconkal.edu.mx). ${ }^{2}$ Unidad de Recursos Naturales-Centro de Investigación Científica de Yucatán. Calle 43 núm. 130 x 32 y 34, Col. Chuburná de Hidalgo, Mérida, Yucatán, México. CP. 97205. (jomijangos@ cicy.mx). Tel. 999 9428330. ${ }^{3}$ Instituto Tecnológico de Tizimín. Carretera Final Aeropuerto Cupul a Tizimín s/n, km 3.5. Tizimín, Yucatán, México. CP. 97000. (pida64@ hotmail.com).

${ }^{\S}$ Autor para correspondencia: renegh10@ hotmail.com.

\section{Resumen}

México es el centro de origen y domesticación del maíz (Zea mays L.), en algunas regiones aún existen poblaciones criollas con potencial para generar variedades mejoradas. El objetivo de este estudio fue evaluar el comportamiento agronómico y fisiológico de doce poblaciones de maíz nativas del sureste de México. El experimento se realizó en Tizimín, Yucatán en el año 2017. Se evaluaron 32 parámetros correspondientes a variables vegetativas, fenológicas, agronómicas y fisiológicas. Los resultados mostraron una diferencia de $75 \mathrm{~cm}$ entre las plantas más bajas (Xn-69) y las de porte más alto (CNB), las plantas de la población DZ-252 presentaron mayor follaje (19.1 hojas) y mayor diámetro de tallo $(26 \mathrm{~mm})$, las poblaciones más precoces fueron CM y CCB (65.5 y 65.8 días, respectivamente). El 58\% de las poblaciones evaluadas superó el rendimiento promedio de la región (4.5 t ha-1). El mayor rendimiento lo obtuvieron las poblaciones CM, Cro, H1 y SP2015 (117.7, 105.8, 111.5 y 109.5 g planta $^{-1}$, respectivamente). Las poblaciones CM, DZ2015, Cja y Cro tuvieron mayor tasa fotosintética; sin embargo, la mayor eficiencia del uso del agua lo presentaron las poblaciones DZ-252 y XnQroo $\left(8.9 \mu \mathrm{mol} \mathrm{CO}_{2} / \mathrm{mmol} \mathrm{H}_{2} \mathrm{O}\right)$. La población CM destacó por su rendimiento y precocidad, la DZ-252 podría utilizarse como forraje por la cantidad de biomasa que generó y la XnQroo debería ser estudiada bajo condiciones de restricción hídrica por su eficiencia del uso del agua, estas tres poblaciones deberían incluirse en programas de mejoramiento genético.

Palabras clave: Zea mays L., diversidad genética, poblaciones criollas.

Recibido: marzo de 2019

Aceptado: julio de 2019 


\section{Introducción}

México es el centro de origen y domesticación del maíz (Zea mays L.) (Matsuoka et al., 2002), en donde se generó gran diversidad de las variedades nativas, como consecuencia de los procesos de selección ejercidos por los pueblos prehispánicos, principales herederos, custodios y mejoradores del germoplasma nativo (Fernández et al., 2013). En México los maíces mejorados satisfacen en buena medida las necesidades de la agroindustria en términos de rendimiento. Sin embargo, 80\% de la superficie de maíz está sembrada con variedades nativas, producidas bajo un sistema de subsistencia o autoconsumo (Sahagún et al., 2008).

Por otra parte, se proyecta que a consecuencia del cambio climático aumentará la temperatura y cambiarán los patrones de precipitación pluvial, que afectará la producción de maíz en muchas regiones de México (Ruiz et al., 2011). Bajo ese escenario, los maíces mejorados podrían ser más afectados; mientras que las nativas que aún conservan sus características genéticas ancestrales podrían ser una alternativa para la producción de maíz a mediano plazo (Hellin et al., 2014). Por lo cual, es importante identificar materiales vegetales con características agronómicas y fisiológicas que favorezcan el desarrollo de nuevas variedades mejoradas (Ramírez-Días et al., 2015).

En este sentido, las poblaciones nativas de maíz que se siembran en condiciones edafo-climáticas particulares del sureste mexicano han sido la base de la alimentación de muchas familias campesinas y por sus características genéticas que aún conservan podrían ser una alternativa para generar nuevas variedades mejoradas (Tuxill et al., 2010); sin embargo, las investigaciones realizadas en esta región se limitan a evaluar el rendimiento y algunas características de grano (Dzib-Aguilar et al., 2011; Cázarez-Sánchez et al., 2015; Coutiño et al., 2015). Por lo tanto, el objetivo de esta investigación fue evaluar el comportamiento agronómico y fisiológico de 12 poblaciones de maíz nativas del sureste de México.

\section{Materiales y métodos}

Se seleccionaron 12 poblaciones de maíces nativos del sureste de México con potencial agronómico, de las cuales nueve (Rocame, Cro; Jarocho, Cja; Morales, CM; Napalú, CNB; Chimbo, CCB; San Pableño, H1; San Pableño, SP2015; Dzit bacal, DZ2015; Xmejen nal, XnQroo) fueron colectadas con productores de la región y tres (Xmejen nal, Xn-69; Dzit bacal, DZ-252; Xmejen nal, Xn-159) de la colección base de maíz del banco de germoplasma del Parque Científico y Tecnológico de Yucatán. Cinco poblaciones provienen de localidades del centro de Chiapas (Cro y Cja, Chiapa de corzo; CM, Villaflores; CNB y CCB, Suchiapa), tres de Campeche (Xn-69, Hopelchén; H1, Becal; SP2015, Vicente Guerrero), dos de Yucatán (DZ-252, Valladolid; DZ2015, Peto) y dos de Quintana Roo (XnQroo, Nueva Reforma; Xn-159, Felipe Carrillo Puerto).

De 12 poblaciones evaluadas cinco son de raza Tuxpeño (Cja, CNB, CCB, H1 y SP2015), dos Vandeño (Cro y CM), tres Nal tel (Xn-69, Xn-159 y XnQro) y dos de Dzit bacal (Dz2015 y Dz252).

El experimento se realizó de febrero-junio de 2017, se uso un sistema de riego del área experimental del Instituto Tecnológico de Tizimín. El clima del sitio es AW0 (cálido subhúmedo con lluvias en verano) (García, 2004). La siembra se realizó por golpe de punzón, con una distancia de $80 \mathrm{~cm}$ entre surcos y $20 \mathrm{~cm}$ entre plantas (62 000 plantas ha-1), el área total sembrada fue de $4608 \mathrm{~m}^{2}$. 
Se aplicó la fórmula 120-80-00 (N-P-K) Kg Ha${ }^{-1}$, la aplicación de fertilizantes se dividió en dos aplicaciones: la mitad del $\mathrm{N}$ y todo el $\mathrm{P}$ se aplicó una semana después de la emergencia de las plántulas, el resto del fertilizante se aplicó cuatro semanas después de la primera aplicación (etapa de crecimiento). El control de malezas se realizó de manera manual y se aplicó sólo en una ocasión Spinetoram para el control de plagas.

\section{Variables evaluadas}

Se evaluaron 32 variables, las cuales se agruparon en cuatro grupos: I) variables vegetativas: altura de planta, número de hojas debajo de mazorca, número de hojas arriba de mazorca, número total de hojas, altura a la mazorca, diámetro del tallo, número total de ramas de la espiga, longitud del pedúnculo de la espiga, longitud del tramo ramificado de la espiga, longitud de la rama central de la espiga y longitud total de la espiga; II) variables fenológicas y de mazorca: días a floración masculina, días a floración femenina, número de mazorcas, diámetro de mazorca, longitud de mazorca, índice diámetro/longitud de mazorca, diámetro de olote y número de hileras de grano; III) variables de rendimiento y grano: peso de grano por planta, peso de 100 granos, longitud de grano, ancho de grano, grosor de grano, índice grosor/longitud de grano, índice grosor/ancho de grano y número de granos por hilera; y IV) variables fisiológicas: fotosíntesis, conductancia estomática, carbono intercelular, transpiración y eficiencia del uso del agua. Las variables de los grupos I, II y III se midieron siguiendo la guía de descriptores para maíz de IBPGR (1991) y el trabajo de Ángeles-Gaspar et al. (2010), para calcular las variables fisiológicas (grupo IV).

Previo a las mediciones puntuales se realizaron mediciones a lo largo del día, donde se determinó que al medio día (12 h) las plantas alcanzaban su máxima tasa fotosintética, además se realizaron curvas de saturación de luz donde se observó que a una densidad de flujo de fotones para la fotosíntesis (DFFF) de $1500 \mu \mathrm{mol} \mathrm{m} \mathrm{m}^{-2} \mathrm{~s}^{-1}$ las plantas de maíz alcanzaban sus valores más altos de fotosíntesis en saturación de luz $\left(\mathrm{A}_{\mathrm{sat}}\right)$. Considerando lo anterior y utilizando un analizador de gases en el infrarrojo (LICOR, LI-6400xt, Nebraska, EE. UU), cuando las plantas se encontraban en fructificación (etapa más demandante de asimilados de carbono) se realizaron mediciones puntuales al medio día, con una DFFF de $1500 \mu \mathrm{mol} \mathrm{m}{ }^{-2} \mathrm{~s}^{-1}$ y una concentración de $\mathrm{CO}_{2}$ de 400 $\mu \mathrm{mol} \mathrm{mol}{ }^{-1}$ (Garruña-Hernández et al., 2014). Las mediciones se realizaron en la parte central de la segunda hoja madura (considerando la hoja bandera como primera), se evaluaron cinco plantas por población, una hoja por planta y se tomaron tres mediciones por hoja.

\section{Diseño experimental y análisis estadístico}

Se utilizó un diseño experimental de bloques completos al azar, con seis repeticiones, cada bloque tenía 24 surcos de $40 \mathrm{~m}$ de largo, cada población de maíz ocupaba dos surcos dentro de cada bloque, la unidad experimental fue de 30 plantas (15 plantas del centro en cada surco); sin embargo, para las variables fisiológicas se midieron cinco plantas por población en la etapa de desarrollo del cultivo (V8-V10). Los datos obtenidos se analizaron con un Andeva y después se realizó una comparación de medias con la prueba de Scott-Knott $(\alpha=0.05)$. Todos los análisis se realizaron con el programa Infostat versión 2016. 


\section{Resultados y discusión}

En las 32 variables evaluadas el análisis de varianza mostró diferencias significativas $(p \leq 0.01)$ entre las poblaciones. Esta variabilidad podría ser explicada por la alta diversidad genética presente en las poblaciones estudiadas, en un trabajo previo Santos et al. (2017) utilizando marcadores moleculares analizaron la diversidad genética de las mismas poblaciones estudiadas en esta investigación y concluyeron que las poblaciones nativas de maíz evaluadas presentan alta diversidad genética $(\mathrm{He}=0.4$ y I= 0.54$)$. Otros trabajos reportan que existe gran variabilidad inter e intra racial entre poblaciones de maíz nativas de la Península de Yucatán (Burgos et al., 2004) y Chiapas (Coutiño et al., 2015), esto podría otorgar una ventaja adaptativa en caso de condiciones adversas (González et al., 2013).

\section{Variables vegetativas}

Respecto a las variables altura de planta (APL) y altura de mazorca (AMZ), las plantas de la población CNB fueron las más altas $(270 \mathrm{~cm})$ y con mazorcas a mayor altura del suelo $(170.4 \mathrm{~cm})$ superando estadísticamente a las demás poblaciones $(p \leq 0.05)$, mientras que las poblaciones XnQroo y Xn-69 fueron las de porte más bajo (197.4 y $195 \mathrm{~cm}$, respectivamente). Entre las plantas de porte más bajo (Xn-69) y las de porte más alto (CNB) se observó una diferencia de $75 \mathrm{~cm}$ (Cuadro 1). Sin embargo, cabe resaltar que la mayoría de las poblaciones estudiadas fueron de porte medio (entre 200 y $240 \mathrm{~cm}$ ), lo cual les confiere tolerancia al acame, la altura de mazorca en la mayoría de estas plantas estuvo entre los 100 y $150 \mathrm{~cm}$, característica altamente deseable para la cosecha (Hernández y Esquivel, 2004).

Cuadro 1. Características vegetativas de poblaciones de maíz nativo del sureste de México (Chiapas, Campeche, Yucatán y Quintana Roo), evaluadas bajo sistema de riego en Tizimín, Yucatán, México.

\begin{tabular}{cccccccccccc}
\hline Gen & $\begin{array}{c}\text { APT } \\
(\mathrm{cm})\end{array}$ & $\begin{array}{c}\text { AMZ } \\
(\mathrm{cm})\end{array}$ & NHBM & NHAM & HT & $\begin{array}{c}\text { DTA } \\
(\mathrm{mm})\end{array}$ & NRE & $\begin{array}{c}\text { LPE } \\
(\mathrm{cm})\end{array}$ & $\begin{array}{c}\text { LTR } \\
(\mathrm{cm})\end{array}$ & $\begin{array}{c}\text { LRC } \\
(\mathrm{cm})\end{array}$ & $\begin{array}{c}\text { LTE } \\
(\mathrm{cm})\end{array}$ \\
\hline CNB & $270 \mathrm{a}$ & $170.4 \mathrm{a}$ & $10 \mathrm{c}$ & $6.8 \mathrm{~b}$ & $16.8 \mathrm{c}$ & $23 \mathrm{~b}$ & $16.5 \mathrm{c}$ & $4.7 \mathrm{~b}$ & $14.8 \mathrm{c}$ & $23.5 \mathrm{c}$ & $38.3 \mathrm{~b}$ \\
DZ2015 & $244.7 \mathrm{~b}$ & $161.5 \mathrm{a}$ & $9.8 \mathrm{c}$ & $7.1 \mathrm{a}$ & $17 \mathrm{c}$ & $23.6 \mathrm{~b}$ & $21.1 \mathrm{~b}$ & $4.7 \mathrm{~b}$ & $16.1 \mathrm{~b}$ & $22.3 \mathrm{c}$ & $38.4 \mathrm{~b}$ \\
Cja & $240.9 \mathrm{~b}$ & $141.6 \mathrm{c}$ & $9.6 \mathrm{c}$ & $7.2 \mathrm{a}$ & $16.8 \mathrm{c}$ & $24.7 \mathrm{a}$ & $17.3 \mathrm{c}$ & $7.1 \mathrm{a}$ & $15.1 \mathrm{c}$ & $26.9 \mathrm{~b}$ & $41.9 \mathrm{a}$ \\
DZ-252 & $240.7 \mathrm{~b}$ & $157.4 \mathrm{~b}$ & $11.9 \mathrm{a}$ & $7.2 \mathrm{a}$ & $19.1 \mathrm{a}$ & $26 \mathrm{a}$ & $23.8 \mathrm{a}$ & $4.4 \mathrm{~b}$ & $18 \mathrm{a}$ & $22.9 \mathrm{c}$ & $40.9 \mathrm{a}$ \\
Xn-159 & $239.5 \mathrm{~b}$ & $160.6 \mathrm{a}$ & $10.4 \mathrm{~b}$ & $7.1 \mathrm{a}$ & $17.6 \mathrm{~b}$ & $22.6 \mathrm{c}$ & $21 \mathrm{~b}$ & $3.9 \mathrm{~b}$ & $14 \mathrm{c}$ & $24.1 \mathrm{~b}$ & $38.1 \mathrm{~b}$ \\
Cro & $237.3 \mathrm{~b}$ & $133.4 \mathrm{c}$ & $8.4 \mathrm{~d}$ & $7.3 \mathrm{a}$ & $15.6 \mathrm{~d}$ & $24 \mathrm{~b}$ & $14.4 \mathrm{~d}$ & $3.6 \mathrm{~b}$ & $15 \mathrm{c}$ & $25.6 \mathrm{~b}$ & $40.6 \mathrm{a}$ \\
$\mathrm{SP} 2015$ & $226.3 \mathrm{~b}$ & $137.6 \mathrm{c}$ & $8.5 \mathrm{~d}$ & $7.2 \mathrm{a}$ & $15.7 \mathrm{~d}$ & $22.3 \mathrm{c}$ & $15.6 \mathrm{c}$ & $3.1 \mathrm{~b}$ & $11.3 \mathrm{~d}$ & $29.7 \mathrm{a}$ & $41 \mathrm{a}$ \\
$\mathrm{CM}$ & $212.4 \mathrm{c}$ & $108.9 \mathrm{e}$ & $7.5 \mathrm{e}$ & $7 \mathrm{a}$ & $14.5 \mathrm{e}$ & $20.3 \mathrm{~d}$ & $12.5 \mathrm{~d}$ & $5.9 \mathrm{a}$ & $10.6 \mathrm{~d}$ & $28.2 \mathrm{a}$ & $38.8 \mathrm{~b}$ \\
$\mathrm{H} 1$ & $207.4 \mathrm{c}$ & $95.2 \mathrm{f}$ & $8.3 \mathrm{~d}$ & $7.3 \mathrm{a}$ & $15.6 \mathrm{~d}$ & $22 \mathrm{c}$ & $13 \mathrm{~d}$ & $4.4 \mathrm{~b}$ & $12.1 \mathrm{~d}$ & $28 \mathrm{a}$ & $40.1 \mathrm{a}$ \\
$\mathrm{CCB}$ & $205 \mathrm{c}$ & $96.8 \mathrm{f}$ & $6.8 \mathrm{e}$ & $7.1 \mathrm{a}$ & $13.9 \mathrm{e}$ & $20.7 \mathrm{~d}$ & $15.7 \mathrm{c}$ & $5.3 \mathrm{a}$ & $14.5 \mathrm{c}$ & $26.9 \mathrm{~b}$ & $41.4 \mathrm{a}$ \\
XnQroo & $197.4 \mathrm{~d}$ & $118.8 \mathrm{~d}$ & $7.5 \mathrm{e}$ & $6.5 \mathrm{~b}$ & $14 \mathrm{e}$ & $20.6 \mathrm{~d}$ & $17.9 \mathrm{c}$ & $6.3 \mathrm{a}$ & $13.6 \mathrm{c}$ & $25.3 \mathrm{~b}$ & $39 \mathrm{~b}$ \\
Xn-69 & $195 \mathrm{~d}$ & $105.7 \mathrm{e}$ & $7.1 \mathrm{e}$ & $7.2 \mathrm{a}$ & $14.3 \mathrm{e}$ & $21.9 \mathrm{c}$ & $19.4 \mathrm{~b}$ & $5.8 \mathrm{a}$ & $12.2 \mathrm{~d}$ & $21.7 \mathrm{c}$ & $34 \mathrm{c}$ \\
\hline
\end{tabular}

Gen (genotipo); APT (altura de planta); AMZ (altura de mazorca); NHBM (núm. de hojas bajo la mazorca); NHAM (núm de hojas arriba de mazorca); HT (hojas totales); DTA (diámetro del tallo); NRE (núm. de ramas de la espiga); LPE (longitud del pedúnculo); LTR (longitud de tramo ramificado); LRC (longitud de rama central); LTE (longitud total de la espiga). Medias con literales diferentes indican diferencias estadísticas significativas (Scott-Knott, $p \leq 0.05$ ). 
Las plantas de la población DZ-252 tuvieron mayor número de hojas totales (19.1), mayor número de hojas bajo la mazorca (11.9) y mayor diámetro de tallo $(26 \mathrm{~mm})$, además se agruparon entre las plantas con mayor número de hojas arriba de la mazorca (7.2) (Cuadro 1). Por otra parte, las plantas de la población XnQroo fueron las menos frondosas, debido a que en las variables número de hojas bajo la mazorca (7.5), número de hojas arriba de la mazorca (6.5), hojas totales (14) y diámetro de tallo $(20.6 \mathrm{~mm})$ se ubicaron en los grupos con valores estadísticamente más bajos (Cuadro 1).

En este sentido, Burgos et al. (2004); Ángeles-Gaspar et al. (2010) mencionan que tanto el número de hojas bajo la mazorca y arriba de la mazorca son parámetros de gran importancia para explicar la diversidad entre poblaciones de maíz. Al respecto, consideramos que son variables altamente importantes; sin embargo, en este trabajo no se podría explicar la diversidad entre las poblaciones considerando sólo estos parámetros, debido a que en el número de hojas bajo la mazorca se forman cinco grupos estadísticamente diferentes, mientras que en el número de hojas arriba de la mazorca solamente se forman dos grupos heterogéneos (uno con 10 poblaciones y otro con dos).

Por otra parte, García y Watson (2003) mencionan una correlación positiva entre el diámetro del tallo y la resistencia al acame en plantas de maíz. Al considerar lo anterior, las plantas de las poblaciones Cja (Chiapas) y DZ-252 (Yucatán) tendrían ventaja ante las demás con relación al acame, como coincidencia las dos poblaciones son de porte medio $(240.9$ y $240.7 \mathrm{~cm})$. En la inflorescencia masculina, las plantas de la población DZ-252 presentaron el mayor número de ramas de la espiga (23.8) y la mayor longitud del tramo ramificado $(18 \mathrm{~cm})$, mientras que las plantas de CM (12.5) y H1 (13) tuvieron la menor cantidad de ramas en la espiga y junto con las plantas de SP2015 los tramos ramificados más cortos $(10.6,12.1$ y $11.3 \mathrm{~cm}$, respectivamente) (Cuadro 1).

En la longitud del pedúnculo las poblaciones formaron dos grupos estadísticos, con excepción de la población Cja $(7.1 \mathrm{~cm})$ las plantas con el pedúnculo más largo (CM: 5.9, CCB: 5.3, XnQroo: 6.3 y Xn-69: $5.8 \mathrm{~cm}$ ) fueron las de menor altura (Cuadro 1). Las plantas de las poblaciones SP2015 $(29.7 \mathrm{~cm}), \mathrm{CM}(28.2 \mathrm{~cm})$ y $\mathrm{H} 1(28 \mathrm{~cm})$ presentaron la rama central más larga y estás mismas poblaciones fueron las que estadísticamente tuvieron el tramo ramificado más corto (Cuadro 1).

En la longitud total de la espiga, se encontraron diferencias estadísticas entre poblaciones, hubo $7.9 \mathrm{~cm}$ de diferencia entre la espiga más larga y la más corta, las plantas de las poblaciones Cja $(41.9 \mathrm{~cm}), \mathrm{CCB}(41.4 \mathrm{~cm}), \mathrm{SP} 2015(41 \mathrm{~cm}), \mathrm{DZ}-252(40.9)$, Cro $(40.6 \mathrm{~cm})$ y H1 $(40.1 \mathrm{~cm})$ presentaron la espiga más larga, mientras que las de la población Xn-69 $(34 \mathrm{~cm})$ la espiga más corta (Cuadro 1). Todas las variables de espiga, resultaron con un coeficiente de variación alto, superior al reportado por Camacho y Chávez (2004). En este sentido, los caracteres de la espiga son de los más importantes para caracterizar variedades y describir la diversidad genética de la región (Burgos et al., 2004).

\section{Variables fenológicas y de mazorca}

Las poblaciones más precoces fueron CM (65.5 y 68.5 días a floración masculina y femenina, respectivamente) y CCB (65.8 y 68.2 días a floración masculina y femenina, respectivamente). En las más precoces la diferencia entre la floración masculina y femenina fue de 3 y 2.4 días (CM y $\mathrm{CCB}$, respectivamente). Las poblaciones más tardías fueron DZ-252 (86.3 y 97 días a floración masculina y femenina, respectivamente) y Xn-159 (85.3 y 93.8 días a floración masculina y femenina) con lapsos entre un evento y otro de 10.7 días (DZ-252) y 8.5 días (Xn-159). 
Las mazorcas de las poblaciones más precoces tuvieron el mayor diámetro (CM: $4.4 \mathrm{~cm}$ y CCB: $4.5 \mathrm{~cm}$ ) y las poblaciones más tardías las mazorcas con menor diámetro (DZ-252: $3.8 \mathrm{~cm}$ y Xn159: $3.9 \mathrm{~cm}$ ) (Cuadro 2). La precocidad es un factor muy importante, que denota la adaptación de las poblaciones a las condiciones ambientales, permite al agricultor elegir que material sembrar según sus necesidades y la época del año (Ángeles-Gaspar et al., 2010). Las plantas de la población DZ-252 fueron las únicas con dos mazorcas por planta, superando estadísticamente a las demás poblaciones que presentaron una mazorca por planta (Cuadro 2).

Cuadro 2. Características fenológicas y de mazorca de poblaciones de maíz nativo del sureste de México (Chiapas, Campeche, Yucatán y Quintana Roo), evaluadas bajo sistema de riego en Tizimín, Yucatán, México.

\begin{tabular}{ccccccccc}
\hline Gen & $\begin{array}{c}\text { DFM } \\
(\text { días })\end{array}$ & $\begin{array}{c}\text { DFF } \\
(\text { días })\end{array}$ & NMZ & $\begin{array}{c}\text { DMZ } \\
(\mathrm{cm})\end{array}$ & $\begin{array}{c}\text { LMZ } \\
(\mathrm{cm})\end{array}$ & DMZ/LMZ & $\begin{array}{c}\text { DOL } \\
(\mathrm{cm})\end{array}$ & NHG \\
\hline CNB & $74.3 \mathrm{c}$ & $89.2 \mathrm{~b}$ & $1.2 \mathrm{c}$ & $3.9 \mathrm{c}$ & $17 \mathrm{a}$ & $0.23 \mathrm{~d}$ & $2.5 \mathrm{~b}$ & $10.7 \mathrm{~d}$ \\
DZ2015 & $82.5 \mathrm{~b}$ & $91 \mathrm{~b}$ & $1.3 \mathrm{~b}$ & $3.3 \mathrm{~d}$ & $14.6 \mathrm{~b}$ & $0.24 \mathrm{~d}$ & $1.9 \mathrm{~d}$ & $10.5 \mathrm{~d}$ \\
$\mathrm{Cja}$ & $72.2 \mathrm{~d}$ & $82 \mathrm{c}$ & $1.3 \mathrm{~b}$ & $4.2 \mathrm{~b}$ & $17.9 \mathrm{a}$ & $0.24 \mathrm{~d}$ & $2.5 \mathrm{~b}$ & $12.4 \mathrm{~b}$ \\
DZ-252 & $86.3 \mathrm{a}$ & $97 \mathrm{a}$ & $1.7 \mathrm{a}$ & $3.8 \mathrm{c}$ & $14.7 \mathrm{~b}$ & $0.27 \mathrm{c}$ & $2.3 \mathrm{c}$ & $12.3 \mathrm{~b}$ \\
Xn-159 & $85.3 \mathrm{a}$ & $93.8 \mathrm{a}$ & $1.5 \mathrm{~b}$ & $3.9 \mathrm{c}$ & $12.9 \mathrm{c}$ & $0.31 \mathrm{~b}$ & $2.2 \mathrm{c}$ & $11.1 \mathrm{~d}$ \\
Cro & $70.8 \mathrm{~d}$ & $77 \mathrm{~d}$ & $1.2 \mathrm{c}$ & $4.3 \mathrm{~b}$ & $17 \mathrm{a}$ & $0.26 \mathrm{c}$ & $2.5 \mathrm{~b}$ & $13.4 \mathrm{~b}$ \\
$\mathrm{SP} 2015$ & $71 \mathrm{~d}$ & $77.3 \mathrm{~d}$ & $1.1 \mathrm{c}$ & $4.2 \mathrm{~b}$ & $16.9 \mathrm{a}$ & $0.25 \mathrm{c}$ & $2.4 \mathrm{~b}$ & $13 \mathrm{~b}$ \\
$\mathrm{CM}$ & $65.5 \mathrm{f}$ & $68.5 \mathrm{f}$ & $1 \mathrm{c}$ & $4.4 \mathrm{a}$ & $16.3 \mathrm{a}$ & $0.27 \mathrm{c}$ & $2.4 \mathrm{~b}$ & $12.9 \mathrm{~b}$ \\
$\mathrm{H} 1$ & $74.8 \mathrm{c}$ & $82.2 \mathrm{c}$ & $1.4 \mathrm{~b}$ & $4.1 \mathrm{~b}$ & $17.3 \mathrm{a}$ & $0.24 \mathrm{~d}$ & $2 \mathrm{~d}$ & $13 \mathrm{~b}$ \\
$\mathrm{CCB}$ & $65.8 \mathrm{f}$ & $68.2 \mathrm{f}$ & $1 \mathrm{c}$ & $4.5 \mathrm{a}$ & $14 \mathrm{~b}$ & $0.33 \mathrm{a}$ & $2.7 \mathrm{a}$ & $12 \mathrm{c}$ \\
XnQroo & $68.2 \mathrm{e}$ & $73.2 \mathrm{e}$ & $1.5 \mathrm{~b}$ & $3.5 \mathrm{~d}$ & $13.2 \mathrm{c}$ & $0.27 \mathrm{c}$ & $1.9 \mathrm{~d}$ & $12.7 \mathrm{~b}$ \\
Xn-69 & $67.8 \mathrm{e}$ & $73 \mathrm{e}$ & $1.4 \mathrm{~b}$ & $4.2 \mathrm{~b}$ & $14.5 \mathrm{~b}$ & $0.29 \mathrm{~b}$ & $2.5 \mathrm{~b}$ & $15.3 \mathrm{a}$ \\
\hline
\end{tabular}

Gen (genotipo); DFM (días a floración masculina); DFF (días a floración femenina); NMZ (núm. mazorcas); DMZ (diámetro mazorca); LMZ (longitud mazorca); DOL (diámetro olote); NHG (núm. hileras de granos). Medias con literales diferentes indican diferencias estadísticas significativas (Scott-Knott, $p \leq 0.05$ ).

En la longitud de la mazorca hay $5 \mathrm{~cm}$ de diferencia entre las poblaciones con la mazorca más larga (Cja: $17.9 \mathrm{~cm})$ y la más corta (Xn-159: $12.9 \mathrm{~cm})($ Cuadro 2). El grupo estadístico con mayor longitud de mazorca se conformó por las poblaciones Cja $(17.9 \mathrm{~cm}), \mathrm{H} 1(17.3 \mathrm{~cm})$, Cro $(17 \mathrm{~cm})$, CNB $(17 \mathrm{~cm}), \mathrm{SP} 2015(16.9 \mathrm{~cm})$ y CM $(16.3 \mathrm{~cm})$, mientras que las poblaciones XnQroo $(13.2 \mathrm{~cm})$ y Xn-159 $(12.9 \mathrm{~cm})$ forman el grupo con mazorcas más cortas (Cuadro 2). Los resultados de diámetro y longitud de mazorca en este trabajo son inferiores a los reportados por Coutiño et al. (2015) en poblaciones de maíz del centro de Chiapas, pero similares a los reportados por Camacho y Chávez (2004) en poblaciones de la Península de Yucatán.

Por otra parte, el índice diámetro/longitud de mazorca es un indicador de la morfometría de la mazorca, un valor alto indica una mazorca más cónica o robusta en la relación ancho-largo. En este sentido, las mazorcas de la población CCB con índice de 0.33 superaron estadísticamente a las de las otras poblaciones, es probable que el grosor del olote tenga influencia sobre esta característica debido a que las mazorcas de CCB también presentaron mayor diámetro de olote $(2.7 \mathrm{~cm})$ superando estadísticamente al resto de las poblaciones (Cuadro 2), el nombre común que recibe esta población es 'Chimbo', cuyo significado hace alusión a la forma ovoide de la mazorca. 
Por otra parte, en la variable hileras de grano, las mazorcas de la población Xn-69 tuvieron más hileras por mazorca (15.3 hileras), superando estadísticamente a las demás poblaciones, mientras que las poblaciones Xn-159 (11.1 hileras), CNB (10.7 hileras) y DZ2015 (10.5 hileras) formaron el grupo estadístico con menos hileras (Cuadro 2).

\section{Variables de rendimiento y grano}

El menor rendimiento (g planta ${ }^{-1}$ ) lo presentaron las dos poblaciones de Yucatán (DZ2015 y DZ-252 con 45.1 y 55.2 g planta $^{-1}$, respectivamente) y una de Quintana Roo (Xn-159 con 56 g planta $^{-1}$ ), el mayor rendimiento de grano por planta lo obtuvieron dos poblaciones provenientes de Chiapas (CM y Cro con 117.7 y $105.8 \mathrm{~g} \mathrm{planta}^{-1}$, respectivamente) y dos provenientes de Campeche (H1 y SP2015 con 111.5 y 109.5 g planta $^{-1}$, respectivamente) (Cuadro 3).

Cuadro 3. Características de rendimiento y grano de poblaciones de maíz nativo del sureste de México (Chiapas, Campeche, Yucatán y Quintana Roo), evaluadas bajo sistema de riego en Tizimín, Yucatán, México.

\begin{tabular}{ccccccccc}
\hline Gen & $\begin{array}{c}\text { RG } \\
\left(\text { g planta }^{-1}\right)\end{array}$ & $\begin{array}{c}\text { P100s } \\
(\mathrm{g})\end{array}$ & $\begin{array}{c}\text { LG } \\
(\mathrm{mm})\end{array}$ & $\begin{array}{c}\text { AG } \\
(\mathrm{mm})\end{array}$ & $\begin{array}{c}\text { GG } \\
(\mathrm{mm})\end{array}$ & GG/LG & GG/AG & GH \\
\hline CNB & $68.2 \mathrm{c}$ & $31.3 \mathrm{~b}$ & $9.5 \mathrm{c}$ & $9.8 \mathrm{a}$ & $4.3 \mathrm{a}$ & $0.5 \mathrm{a}$ & $0.5 \mathrm{a}$ & $25.7 \mathrm{c}$ \\
DZ2015 & $45.1 \mathrm{~d}$ & $22.7 \mathrm{f}$ & $9.7 \mathrm{c}$ & $8.7 \mathrm{c}$ & $3.3 \mathrm{~d}$ & $0.4 \mathrm{c}$ & $0.4 \mathrm{~b}$ & $24.5 \mathrm{c}$ \\
Cja & $86.7 \mathrm{~b}$ & $29.4 \mathrm{c}$ & $10.4 \mathrm{~b}$ & $9.3 \mathrm{~b}$ & $3.9 \mathrm{~b}$ & $0.5 \mathrm{~b}$ & $0.4 \mathrm{a}$ & $31 \mathrm{~b}$ \\
DZ-252 & $55.2 \mathrm{~d}$ & $23.6 \mathrm{e}$ & $9.9 \mathrm{c}$ & $9 \mathrm{c}$ & $3.8 \mathrm{~b}$ & $0.4 \mathrm{~b}$ & $0.4 \mathrm{a}$ & $23.5 \mathrm{c}$ \\
Xn-159 & $56 \mathrm{~d}$ & $23.9 \mathrm{e}$ & $9.8 \mathrm{c}$ & $9 \mathrm{c}$ & $3.5 \mathrm{c}$ & $0.4 \mathrm{c}$ & $0.4 \mathrm{~b}$ & $24.1 \mathrm{c}$ \\
Cro & $105.8 \mathrm{a}$ & $30.7 \mathrm{~b}$ & $10.4 \mathrm{~b}$ & $8.9 \mathrm{c}$ & $3.8 \mathrm{~b}$ & $0.4 \mathrm{c}$ & $0.4 \mathrm{a}$ & $32.9 \mathrm{a}$ \\
SP2015 & $109.5 \mathrm{a}$ & $28.4 \mathrm{~d}$ & $10.9 \mathrm{~b}$ & $8.8 \mathrm{c}$ & $3.8 \mathrm{~b}$ & $0.4 \mathrm{c}$ & $0.4 \mathrm{a}$ & $32.3 \mathrm{a}$ \\
CM & $117.7 \mathrm{a}$ & $29.5 \mathrm{c}$ & $11.6 \mathrm{a}$ & $9.4 \mathrm{~b}$ & $3.7 \mathrm{~b}$ & $0.3 \mathrm{~d}$ & $0.4 \mathrm{~b}$ & $33.5 \mathrm{a}$ \\
H1 & $111.5 \mathrm{a}$ & $30 \mathrm{c}$ & $11.3 \mathrm{a}$ & $8.5 \mathrm{~d}$ & $3.8 \mathrm{~b}$ & $0.3 \mathrm{~d}$ & $0.4 \mathrm{a}$ & $34.7 \mathrm{a}$ \\
CCB & $95.1 \mathrm{~b}$ & $33 \mathrm{a}$ & $10.4 \mathrm{~b}$ & $10.1 \mathrm{a}$ & $3.4 \mathrm{~b}$ & $0.5 \mathrm{~b}$ & $0.4 \mathrm{~b}$ & $26 \mathrm{c}$ \\
XnQroo & $82.7 \mathrm{~b}$ & $18.9 \mathrm{~g}$ & $9.6 \mathrm{c}$ & $7.5 \mathrm{e}$ & $3.2 \mathrm{~d}$ & $0.3 \mathrm{~d}$ & $0.4 \mathrm{a}$ & $29.6 \mathrm{~b}$ \\
Xn-69 & $66.5 \mathrm{c}$ & $23.2 \mathrm{f}$ & $9.9 \mathrm{c}$ & $8.3 \mathrm{~d}$ & $3.8 \mathrm{~b}$ & $0.5 \mathrm{~b}$ & $0.5 \mathrm{a}$ & $26.8 \mathrm{c}$ \\
\hline
\end{tabular}

Gen (genotipo); RG (rendimiento de grano); P100s (peso de 100 semillas); LG (longitud de grano); AG (ancho de grano); GG (grosor de grano); GH (núm. granos por hilera). Medias con literales diferentes indican diferencias estadísticas significativas (Scott-Knott, $p \leq 0.05$ ).

Sin embargo, ninguna de estas cuatro poblaciones con mayor rendimiento destacó en la variable 'peso de 100 semillas', donde la población que superó estadísticamente a las demás fue la CCB (33 g), también proveniente de Chiapas (Cuadro 3). Esto indica que las poblaciones con mayor rendimiento no tenían los granos más pesados ni más grandes, pues a pesar de que los granos de CM y H1 fueron los más largos (11.6 y $11.3 \mathrm{~mm}$, respectivamente), los granos de las cuatro poblaciones con mayor rendimiento no destacaron en ancho y grosor, en esas variables destacó la población CNB (con 9.8 y $4.3 \mathrm{~mm}$, respectivamente); por lo tanto, la población CNB superó estadísticamente a todas las demás en el índice grosor/longitud de grano (0.5). 
Cabe resaltar, que las cuatro poblaciones con mayor rendimiento fueron las que presentaron mayor número de granos por hileras (H1: 34.7, CM: 33.5, Cro: 32.9 y SP2015: 32.3 granos/hilera), mientras que las mazorcas de la población CCB que habían obtenido los granos más pesados (con base en el peso de 100 semillas) estadísticamente se ubicaron en el grupo con menos granos por hilera (Xn-69: 26.8, CCB: 26, CNB: 25.7, DZ2015: 24.5, Xn-159: 24.1, DZ-252: 23.5 granos/hilera) (Cuadro 3). Estos resultados demuestran que las poblaciones provenientes de otros sitios del sureste de México pueden superar en rendimiento a las poblaciones que por años han utilizado los agricultores de la región, que puede ser una alternativa de siembra debido al potencial que tienen para convertirse en variedades mejoradas.

Si se considera que el rendimiento de maíz de riego en el sureste mexicano es de $4.5 \mathrm{t} \mathrm{ha}^{-1}$ (SIAP, 2016), 58\% de las poblaciones evaluadas superan el rendimiento promedio sugerido para la región, destacando las $7.3 \mathrm{t} \mathrm{ha}^{-1}$ de la población CM de Chiapas, equivalente al híbrido (H-520), uno de los más recomendados para la región (Sierra-Macías et al., 2008).

Además, en este estudio el número de mazorcas por planta no influyó en el rendimiento final de producción, pues la única población que presentó dos mazorcas por planta (DZ-252) fue de las menos productivas. De esta manera, se demuestra que el potencial que tienen algunas poblaciones nativas del sureste de México puede contribuir para el mejoramiento genético en la región del trópico mexicano (Arellano et al., 2010).

\section{Variables fisiológicas}

En general las plantas de todas las poblaciones tuvieron valores altos de asimilación neta (fotosíntesis) y tasas bajas de carbono intercelular (Cuadro 4), el hecho de contar con metabolismo C4 les confiere esta cualidad. Sin embargo, en la tasa de asimilación neta (fotosíntesis) las poblaciones CM $\left(39.8 \mu \mathrm{mol} \mathrm{m} \mathrm{s}^{-2}\right)$, DZ2015 $\left(37.8 \mu \mathrm{mol} \mathrm{m} \mathrm{m}^{-2} \mathrm{~s}^{-1}\right)$, Cja $\left(37.3 \mu \mathrm{mol} \mathrm{m} \mathrm{m}^{-2}\right)$ y Cro $\left(37.2 \mu \mathrm{mol} \mathrm{m} \mathrm{m}^{-2} \mathrm{~s}^{-1}\right)$ superaron estadísticamente a las demás (Cuadro 4). Cong-feng et al. (2015) mencionan que la capacidad fotosintética está relacionada con el rendimiento de grano y la producción de biomasa.

Cuadro 4. Características fisiológicas de poblaciones de maíz nativo del sureste de México (Chiapas, Campeche, Yucatán y Quintana Roo), evaluadas bajo sistema de riego en Tizimín, Yucatán, México.

\begin{tabular}{cccccc}
\hline Gen & $\begin{array}{c}\mathrm{A}_{\mathrm{N}} \\
\left(\mu \mathrm{mol} \mathrm{m}^{-2} \mathrm{~s}^{-1}\right)\end{array}$ & $\begin{array}{c}\mathrm{g}_{\mathrm{s}} \\
\left(\mu \mathrm{mol} \mathrm{m}^{-2} \mathrm{~s}^{-1}\right)\end{array}$ & $\begin{array}{c}\mathrm{C}_{\mathrm{i}} \\
\left(\mu \mathrm{mol} \mathrm{mol}^{-1}\right)\end{array}$ & $\begin{array}{c}\mathrm{E} \\
\left(\mathrm{mmol} \mathrm{m}^{-2} \mathrm{~s}^{-1}\right)\end{array}$ & $\begin{array}{c}\mathrm{EUA} \\
\left(\mu \mathrm{mol} \mathrm{CO} / \mathrm{mmol} \mathrm{H}_{2} \mathrm{O}\right)\end{array}$ \\
\hline $\mathrm{CNB}$ & $35.3 \mathrm{~b}$ & $0.29 \mathrm{~b}$ & $133.0 \mathrm{a}$ & $5.2 \mathrm{~b}$ & $6.8 \mathrm{e}$ \\
$\mathrm{DZ2015}$ & $37.8 \mathrm{a}$ & $0.28 \mathrm{~b}$ & $109.2 \mathrm{c}$ & $4.4 \mathrm{c}$ & $8.6 \mathrm{~b}$ \\
$\mathrm{Cja}$ & $37.3 \mathrm{a}$ & $0.27 \mathrm{~b}$ & $94.4 \mathrm{~d}$ & $5.9 \mathrm{a}$ & $6.4 \mathrm{f}$ \\
$\mathrm{DZ}-252$ & $34.5 \mathrm{~b}$ & $0.27 \mathrm{~b}$ & $123.9 \mathrm{~b}$ & $3.9 \mathrm{c}$ & $8.9 \mathrm{a}$ \\
Xn-159 & $34.5 \mathrm{~b}$ & $0.24 \mathrm{c}$ & $92.9 \mathrm{~d}$ & $5.2 \mathrm{~b}$ & $6.7 \mathrm{e}$ \\
Cro & $37.2 \mathrm{a}$ & $0.28 \mathrm{~b}$ & $106.6 \mathrm{c}$ & $4.3 \mathrm{c}$ & $8.7 \mathrm{~b}$ \\
$\mathrm{SP} 2015$ & $36.2 \mathrm{~b}$ & $0.25 \mathrm{c}$ & $98.3 \mathrm{~d}$ & $5.1 \mathrm{~b}$ & $7.1 \mathrm{~d}$ \\
$\mathrm{CM}$ & $39.8 \mathrm{a}$ & $0.34^{\mathrm{a}}$ & $137.8 \mathrm{a}$ & $5.7 \mathrm{a}$ & $6.9 \mathrm{~d}$ \\
\hline
\end{tabular}




\begin{tabular}{cccccc}
\hline Gen & $\begin{array}{c}\mathrm{A}_{\mathrm{N}} \\
\left(\mu \mathrm{mol} \mathrm{m}^{-2} \mathrm{~s}^{-1}\right)\end{array}$ & $\begin{array}{c}\mathrm{g}_{\mathrm{s}} \\
\left(\mu \mathrm{mol} \mathrm{m}^{-2} \mathrm{~s}^{-1}\right)\end{array}$ & $\begin{array}{c}\mathrm{C}_{\mathrm{i}} \\
\left(\mu \mathrm{mol} \mathrm{mol}^{-1}\right)\end{array}$ & $\begin{array}{c}\mathrm{E} \\
\left(\mathrm{mmol} \mathrm{m}^{-2} \mathrm{~s}^{-1}\right)\end{array}$ & $\begin{array}{c}\mathrm{EUA} \\
\left(\mu \mathrm{mol} \mathrm{CO} / \mathrm{mmol} \mathrm{H}_{2} \mathrm{O}\right)\end{array}$ \\
\hline $\mathrm{H} 1$ & $35.8 \mathrm{~b}$ & $0.24 \mathrm{c}$ & $92.8 \mathrm{~d}$ & $4.9 \mathrm{~b}$ & $7.4 \mathrm{c}$ \\
$\mathrm{CCB}$ & $35.3 \mathrm{~b}$ & $0.23 \mathrm{c}$ & $83.2 \mathrm{~d}$ & $5 \mathrm{~b}$ & $7.1 \mathrm{~d}$ \\
XnQroo & $35.5 \mathrm{~b}$ & $0.26 \mathrm{c}$ & $111.1 \mathrm{c}$ & $4 \mathrm{c}$ & $8.9 \mathrm{a}$ \\
Xn-69 & $35.7 \mathrm{~b}$ & $0.24 \mathrm{c}$ & $92.5 \mathrm{~d}$ & $4.1 \mathrm{c}$ & $8.7 \mathrm{~b}$ \\
\hline
\end{tabular}

Gen (genotipo); $\mathrm{A}_{\mathrm{N}}$ (fotosíntesis); $\mathrm{g}_{\mathrm{s}}$ (conductancia estomática); $\mathrm{C}_{\mathrm{i}}$ (carbono intercelular); E (transpiración), EUA (eficiencia de uso del agua). Medias con literales diferentes indican diferencias estadísticas (Scott-Knott, $p \leq 0.05$ ).

En este sentido, las plantas de las poblaciones DZ2015 y Cja fueron de las más altas. Las cuatro poblaciones con mayor tasa fotosintética (CM, DZ2015, Cja y Cro) fueron de las que más hojas acumularon por debajo de la mazorca, y Cja, Cro y CM fueron de las que tuvieron mazorcas más largas, esto sugiere que la mayor cantidad de los asimilados de carbono los derivaron a producción de biomasa, específicamente para incrementar altura y zona basal de planta, así como también tamaño de mazorca.

Asimismo, de las cuatro poblaciones destacadas en rendimiento, las plantas de las poblaciones CM y Cro sobresalieron, cabe mencionar que las poblaciones con alto rendimiento eran de porte medio (de 207 a $237 \mathrm{~cm}$ ), lo cual sugiere que gran parte de sus fotoasimilados los destinaron para incrementar el número de granos por hilera y así aumentar su rendimiento. Las plantas de la población CM además de ser las que presentaron la tasa de fotosíntesis más alta, también tuvieron la mayor conductancia estomática $\left(0.34 \mu \mathrm{mol} \mathrm{m} \mathrm{m}^{-2} \mathrm{~s}^{-1}\right)$.

El (Cuadro 4) tuvieron el mayor intercambio gaseoso $\left(\mathrm{CO}_{2}\right.$ y $\left.\mathrm{H}_{2} \mathrm{O}\right)$ entre el interior de las hojas y la atmosfera circundante, esto ocasionó que su tasa de transpiración $\left(5.7 \mathrm{mmol} \mathrm{m}^{-2} \mathrm{~s}^{-1}\right)$ fuera una de las más altas (Cuadro 4) y la pérdida de agua a través de los estomas durante el intercambio de gases influyó para que la eficiencia del uso del agua en las plantas de CM haya sido de las más bajas $\left(6.9 \mu \mathrm{mol} \mathrm{CO} / \mathrm{CO}_{2} \mathrm{H}_{2} \mathrm{O}\right)$ (Cuadro 4). Con base en lo mencionado, $\mathrm{CM}$ fue la población con mejor rendimiento, para asegurar su excelente productividad (rendimiento de grano) se recomienda sembrar bajo condiciones de riego y no de temporal.

Como se mencionó anteriormente las plantas de la población DZ-252 fueron las más frondosas (mayor número de hojas y tallos más gruesos), a pesar de tener la tasa de fotosíntesis más baja $\left(34.5 \mu \mathrm{mol} \mathrm{m} \mathrm{m}^{-2} \mathrm{~s}^{-1}\right)$ también tuvieron la tasa de transpiración más baja, esto indica que fijan menos carbono que las demás poblaciones pero al mismo tiempo pierden menos agua durante el intercambio de gases, lo que ocasionó que junto con la población XnQroo tuvieran la mejor eficiencia del uso del agua $\left(8.9 \mu \mathrm{mol} \mathrm{CO}_{2} / \mathrm{mmol} \mathrm{H}_{2} \mathrm{O}\right)$, además, biológicamente emplean un mecanismo de reproducción y sobrevivencia pues producen dos mazorcas en lugar de una.

Cabe mencionar, que esta población (DZ-252) proviene de la misma región donde se llevó a cabo el estudio y los resultados tanto agronómicos como fisiológicos demostraron la adaptación que existe a las condiciones ambientales del lugar. Yang et al. (2012) mencionan que valores altos en la eficiencia del uso del agua es característico de plantas adaptadas a condiciones de sequía. Las plantas de DZ-252 no tienen un buen rendimiento de grano en comparación con otras poblaciones evaluadas en este estudio, pero tienen un excelente desempeño en la acumulación de biomasa y la 
mejor eficiencia del uso del agua, se podría recomendar su uso como forraje o como producción de grano en temporal, esto último esperando rendimientos bajos, pero con la posibilidad de que fisiológicamente pueda soportar eventos de sequía intraestival.

Además, se podría incluir en programas de mejoramiento genético para buscar resistencia a sequía junto con poblaciones con buen rendimiento.

\section{Conclusiones}

Los cuatro grupos de variables ayudaron a resaltar la diversidad genética que existe entre las poblaciones. A nivel morfológico tanto en planta como en mazorca hubo una alta variabilidad. Las poblaciones de maíz provenientes de otras regiones pueden desarrollarse igual o mejor que las cultivadas comúnmente en la región. El mayor rendimiento de grano lo obtuvieron dos poblaciones provenientes de Chiapas (CM y Cro) y dos provenientes de Campeche (H1 y SP2015).

El menor rendimiento lo tuvieron dos poblaciones de Yucatán (DZ2015 y DZ-252) y una de Quintana Roo (Xn-159). La población CM proveniente de Chiapas destacó por su rendimiento y precocidad, es un excelente material que podría ser utilizado en la generación de variedades mejoradas; sin embargo, se recomienda su siembra en condiciones de riego, debido a la alta pérdida de agua (transpiración) que tiene durante el intercambio de gases (fotosíntesis).

Por la eficiencia del uso del agua (fotosíntesis/transpiración) la población DZ-252 se podría utilizar como forraje, debido a la elevada producción de biomasa que presenta y la población XnQroo como producción de grano en temporal, ambos materiales, podrían soportar eventos de sequía intraestival y garantizar el sustento de los agricultores que no cuentan con sistema de riego.

\section{Agradecimientos}

Se agradece al CONACYT por la beca número 572785 otorgada para estudios de posgrado del primer autor, también al Instituto Tecnológico de Tizimín por la disponibilidad para el establecimiento y desarrollo del experimento.

\section{Literatura citada}

Ángeles, G. E.; Ortiz, T. E.; López, P. E. y López, R. G. 2010. Caracterización y rendimiento de poblaciones de maíz nativas de Molcaxac, Puebla. Rev. Fitotec. Mex. 33(4):287-296.

Arellano, V. J. L.; Gámez, V. A. J. y Ávila, P. M. A. 2010. Potencial agronómico de variedades criollas de maíz Cacahuacintle en el valle de Toluca. Rev. Fitotec. Mex. 33(4):37-41.

Burgos, M. L. A.; Chávez, S. J. L. y Ortíz, C. J. 2004. Variabilidad morfológica de maíces criollos de la Península de Yucatán, México: In: manejo de la diversidad de los cultivos en los agroecosistemas tradicionales. Chávez, S. J. L.; Tuxill, J. y Jarvis, D. I. (Eds.). Instituto Internacional de Recursos Fitogenéticos. Cali, Colombia. 58-66 pp.

Camacho, V. T. C. y Chávez, S. J. L. 2004. Diversidad morfológica del maíz criollo de la región centro de Yucatán, México. In: manejo de la diversidad de los cultivos en los agroecosistemas tradicionales. Chávez, S. J. L.; Tuxill, J. y Jarvis, D. I. (Eds.). Instituto Internacional de Recursos Fitogenéticos. Cali, Colombia. 47-57 pp. 
Cázarez, S. E.; Chávez, S. J. L.; Salinas, M. Y.; Castillo, G. F. y Ramírez, V. P. 2015. Variación en la composición del grano entre poblaciones de maíz (Zea mays L.) nativas de Yucatán, México. Agrociencia. 49(1):15-30.

Cong, F. L.; Zhi, Q. T.; Peng, L.; Ji, W. Z.; Ke, Z. Z.; Shu, T. D. and Ming, Z. 2015. Increased grain yield with improved photosynthetic characters in modern maize parental lines. J. Integrative Agric. 14(9):1735-1744.

Coutiño, E. B.; Vidal, M. V. A.; Cruz, V. C. y Gómez G. M. 2015. Características eloteras y de grano de variedades nativas de maíz de Chiapas. Rev. Mex. Cienc. Agríc. 5(6):1119-1127.

Dzib, A. L. A.; Segura, C. J. C.; Ortega, P. R. y Latournerie, M. L. 2011. Cruzas dialélicas entre poblaciones nativas de maíz de Yucatán y poblaciones mejoradas. Trop. Subtrop. Agroecosys. 14(1):119-127.

Fernández S. R.; Morales, C. L. A. y Gálvez, M. A. 2013. Importancia de los maíces nativos de México en dieta nacional. Revisión indispensable. Rev. Fitotec. Mex. 36(3-A):275-283.

García, E. 2004. Modificaciones al sistema de clasificación climática de Köppen. 5ª (Ed.). Comisión Nacional para el Estudio de la Biodiversidad (CONABIO). DF, México. 41 p.

García. M. y Watson, C. E. Jr. 2003. Herencia de la resistencia al acame de raíces en maíz dulce (Zea mays L.). Revista UDO Agrícola. 3(1):24-33.

Garruña, H. R.; Orellana, R.; Larqué, S. A. and Canto, A. 2014. Understanding the physiological responses of a tropical crop (Capsicum chinense Jacq.) at high temperature. PLoS ONE 9(11): e111402. doi:10.1371/journal.pone.0111402.

González, C. M. E.; Palacios, N. R.; Espinoza, A. B. y Bedoya, C. A. S. 2013. Diversidad genética en maíces nativos mexicanos tropicales. Rev. Fitotec. Mex. 36(3-A):329-338.

Hellin, J.; Bellon, M. R. and Hearne, S. J. 2014. Maize landraces and adaptation to climate change in Mexico. J. Crop Improvement. 28(4):484-501.

Hernández, C. J. M. y Esquivel E. G. 2004. Rendimiento de grano y características agronómicas en germoplasma de maíz de Valles Altos de México. Rev. Fitotec. Mex. 27(1):27-31.

IBPGR. 1991. International Board for Plant Genetic Resources. Descriptors for maize. International maize and wheat improvement center-international board for plant genetic Resources. Rome. 87 p.

Matsuoka, Y.; Vigouroux, Y.; Goodman, M. M.; Sanchez, G. J.; Buckler, E. and Doebley, J. 2002. A single domestication for maize shown by multilocus microsatellite genotyping. Proceedings of the National Academy of Sciences. 99(9):6080-6084.

Ramírez, D. J. L.; Ledesma, M. A.; Vidal, M. V. A.; Gómez, M. N. O.; Ruiz, C. J. A.; Velázquez, C. G. A.; Ron, P. J.; Salinas, M. Y. y Nájera, C. L. A. 2015. Selección de maíces nativos como donadores de características agronómicas útiles en híbridos comerciales. Rev. Fitotec. Mex. 38(2):119-131.

Ruiz, C. J. A.; Medina, G. G.; Ramírez, D. J. L.; Flores, L. H. E.; Ramírez, O. G.; Manríquez, O. J. D.; Zarazúa, V. P.; González, E. D. R.; Días, P. D. y Mora, O. C. 2011. Cambio climático y sus implicaciones en cinco zonas productoras de maíz en México. Rev. Mex. Cienc. Agríc. 2(2):309-323.

Sahagún, C. L.; Barrera, G. E.; Márquez, S. F.; Gómez, M. N.; Trujillo, C. A.; Castro, G. H.; Coutiño, E. B.; Carrera, V. J. A.; Castellanos, S. A. y Luévano, V. J. M. 2008. Evaluación de variedades de maíz del CENREMMAC mejoradas por retrocruza limitada para resistencia a sequía. Rev. Geografía Agrícola. 40:90-100.

Santos F. C. L.; Andueza, N. R. H.; Ruíz S. E.; Latournerie, M. L.; Garruña R.; Mijangos, C. J. O.; Martínez, C. J. 2017. Characterization of the genetic structure and diversity of maize (Zea mays L.) landrace populations from Mexico. Maydica. 62(2):1-7. 
SIAP. 2016. Servicio de Información Agroalimentaria y Pesquera. Datos de producción agrícola. Resumen Nacional por estado. http://www.gob.mx/siap/.

Sierra, M. M.; Palafox, C. A.; Rodríguez, M. F.; Espinosa, C. A.; Gómez, M. N.; Caballero, H. F.; Barrón, F. S.; Zambada, M. A. y Vázquez, C. G. 2008. H-520, híbrido trilineal de maíz para el trópico húmedo de México. Agric. Téc. Méx. 34(1):119-122.

Tuxill, J.; Arias, R. L.; Latournerie, M. L. and Cob, U. V. 2010. All maize is not equal: maize variety choices and mayan foodways in rural Yucatan, Mexico. In: Staller, J. E. and Carrasco, M. (Eds.) Pre-Columbian foodways interdisciplinary approaches to food, culture, and markets in ancient Mesoamerica. Springer Science+Business Media New York, USA. $477 \mathrm{p}$.

Yang, Z.; Sinclair, T. R.; Zhu, M.; Messina, C. D. and Cooper, M. 2012. Temperature effect on transpiration response of maize plants to vapour pressure deficit. Environ. Exp. Bot. 78(4):157-162. 